\title{
Semiconductor Devices for All Optical Signal Processing: Just How Fast can They Go?
}

Mørk, Jesper; Mecozzi, A

Published in:

1999 IEEE LEOS Annual Meeting Conference Proceedings

Link to article, DOI:

10.1109/LEOS.1999.812026

Publication date:

1999

Document Version

Publisher's PDF, also known as Version of record

Link back to DTU Orbit

Citation (APA):

Mørk, J., \& Mecozzi, A. (1999). Semiconductor Devices for All Optical Signal Processing: Just How Fast can They Go? In 1999 IEEE LEOS Annual Meeting Conference Proceedings (Vol. 2, pp. 900-901). IEEE. https://doi.org/10.1109/LEOS.1999.812026

\section{General rights}

Copyright and moral rights for the publications made accessible in the public portal are retained by the authors and/or other copyright owners and it is a condition of accessing publications that users recognise and abide by the legal requirements associated with these rights.

- Users may download and print one copy of any publication from the public portal for the purpose of private study or research.

- You may not further distribute the material or use it for any profit-making activity or commercial gain

- You may freely distribute the URL identifying the publication in the public portal 


\title{
Semiconductor Devices for All-Optical Signal Processing: Just How Fast Can They Go?
}

\author{
J. Mørk \\ A. Mecozzi \\ Fondazione Ugo Bordoni \\ via B. Castiglione 59, Rome Italy
}

Research Center COM, Technical University of Denmark

Build. 349, DK-2800 Lyngby, Denmark.E-mail: jm@com.dtu.dk

The development of devices that can accomplish all-optical signal processing is important in order to meet the demand for bandwidth and flexibility in communication networks. In wavelength-division multiplexed (WDM) systems, the signal processing elements, for separating wavelength channels, e.g., are mainly passive. In systems based on optical-time-division multiplexing (OTDM) of trains of short optical pulses, active devices are required. For instance, optical switches that can gate out a single channel before electronic detection or that can perform add-drop operations at network nodes are required [1]. Several different semiconductor device structures for accomplishing all-optical signal processing have been proposed, but they nearly all employ the semiconductor optical amplifier (SOA) as a central element. In this talk we will discuss the physical processes in SOA's that are important in determining the speed of SOA based switches. We shall consider both devices based on incoherent processes, such as optically induced cross-gain and cross-phase modulation as well as devices employing coherent four-wave mixing.

Travelling wave effects have been realized to be important in understanding the frequency response of wavelength conversion based on cross-gain or cross-phase modulation in SOAs [2]. Thus, due to the saturation characteristics of the SOA gain, the wavelength-converted signals are high-frequency filtered and this increases the bandwidth beyond what is expected from the value of the stimulated carrier lifetime [2,3]. This effect has been studied also for the case of cascaded amplifiers and shown to be strongly influenced by the value of the loss in-between the amplifiers [4]. A very similar effect leads to an unexpected "resonance-like" peak in the current-modulation response of SOAs [5].

Nonlinear gain effects due to carrier heating and spectral holeburning lead to additional, ultra-fast, saturation of the SOA gain beyond the carrier density induced changes and therefore further increase the bandwidth of cross-gain based SOA switches [6]. Considering the saturation of SOAs due to single-pulse amplification, it was shown experimentally and theoretically, that for pulses shorter than approximately $10 \mathrm{ps,} \mathrm{ultra-fast} \mathrm{carrier} \mathrm{dynamics} \mathrm{give} \mathrm{the} \mathrm{main} \mathrm{contribution} \mathrm{to}$ gain saturation [7]. This shows that for trains of very short pulses, the SOA dynamics may be very different and one may envisage other ways of extending the bandwidth.

Smart ways of getting rid of the slow carrier density recovery by using delay-differential techniques have been demonstrated [8-10]. These techniques use that in interferometrically based 
devices, it is the phase difference between the arms of the interferometer that determine the interferometer output. By using two control pulses, which can be implemented in various ways [8-10], one can null out the phase difference after a controllable time-delay. The bit-rate of the constituent OTDM channels, rather than the aggregate bit-rate, limits the speed of the device, since the carrier density needs time to recover in-between the turn-on events. In this delaydifferential case, ultra-fast refractive index dynamics may degrade the performance, leading to non-flat switching windows.

Four-wave mixing (FWM) relies on coherent beating between two beams, and - not being limited in speed directly by the time constants of the SOA - is often referred to as being a bit-rate transparent technique. For large wavelength detuning, as required for wavelength conversion and de-multiplexing of short pulses, the efficiency of the FWM process drops and this limits the signal to noise ratio. Also, pattern effects may pose a problem for very high bit-rates due to residual gain modulation [11]. The saturation characteristics of the medium implies that the optimum operation point still depends on bit-rate and pulsewidth [12-13]. Still, however, this is a promising technique for all-optical processing of signals at very high bit-rates.

[1] S. Kawanishi, IEEE J. Quantum Electron., vol. 34, pp. 2064, 1998.

[2] C. Joergensen et al., IEEE J. Select. Topics Quantum Electron., vol. 3, pp. 1168, Oct. 1997.

[3] A. Mecozzi, IEEE Photon. Technol. Lett., vol. 8, pp. 1471, 1996.

[4] D. Marcenac and A. Mecozzi, IEEE Photon.\Technol.\Lett., vol. 9, pp. 749, June 1997.

[5] J. Mørk, A. Mecozzi and G. Eisenstein, to appear in IEEE J. Select. Topics Quantum Electron

[6] D. Marcenac et al., in Proc. COST239/240 Workshop on Semiconductor Optical

Amplifiers, Prague, 27-28 October 1997.

[7] P. Borri et al., Opt. Commun., vol. 164, pp. 51, 1999.

[8] K. Suzuki et al., Electron. Lett., vol. 30, pp. 1501, 1994.

[9] E. Jahn et al., Electron. Lett., vol. 31, pp. 1857, 1995.

[10] K.L. Hall and K.A. Rauschenbach, Opt. Lett., vol. 23, pp. 1271, 1998.

[11] M. Shtaif and G. Eisenstein, J. Lightwave Technol., vol. 14, pp. 2069, 1996.

[12] S. Diez et al., IEEE J. Select. Topics Quantum Electron., vol. 3, pp. 1131, 1997.

[13] A. Mecozzi and J. Mørk, IEEE J. Select. Topics Quantum Electron., vol. 3, pp. 1190, 1997. 